\title{
Is Laparoscopic Approach Also Safe for the Treatment of Remnant Gastric Cancer?
}

\author{
Kyo Young Song, M.D., Ph.D., FACS \\ Department of Surgery, College of Medicine, The Catholic University of Korea, Seoul, Korea
}

\begin{abstract}
The most important advantages of laparoscopic gastrectomy are the minimal invasiveness, including less postoperative pain, shorter recovery, and minimal complications. A laparoscopic distal gastrectomy is accepted widely as a standard treatment for gastric cancer. On the other hand, a laparoscopic total gastrectomy has not been popularized as a distal gastrectomy because of the complexity of a lymph node dissection and the diversity of reconstruction. In terms of laparoscopic surgery for a remnant gastrectomy, there are three key points, which are critical for safe operation: adequate lymph node dissection, meticulous adhesiolysis, and reconstruction. After radical surgery for gastric cancer, the intra-abdominal condition is greatly changed. In addition, the lymphatic anatomy around the stomach is broken and surgeons should be aware of a newly developed lymphatic system to perform adequate node dissection. An esophago-jejunal reconstruction is at risk of leakage. Until evidence that is more concrete can be obtained, experienced surgeons should consider the laparoscopic approach.
\end{abstract}

Keywords: Gastrectomy, Gastric cancer, Gastric remnant, Laparoscopy, Lymph node dissection
Received February 8, 2019

Revised February 27, 2019

Accepted March 5, 2019

Corresponding author

Kyo Young Song

Department of Surgery, Seoul St.

Mary's Hospital, The Catholic

University of Korea, 222 Banpo-

daero, Seocho-gu, Seoul 06591,

Korea

Tel: +82-2-2258-6601

Fax: +82-2-595-2822

E-mail:skygs@catholic.ac.kr

ORCID:

http://orcid.org/0000-0002-5840-1638

This is an Open Access article distributed under the terms of the Creative Commons Attribution Non-Commercial License (http:// creativecommons.org/licenses/by-nc/4.0/) which permits unrestricted non-commercial use, distribution, and reproduction in any medium, provided the original work is properly cited.

Copyright (c) 2019 The Journal of Minimally Invasive Surgery. All rights reserved.
Since the introduction of laparoscopic gastrectomy by $\mathrm{Ki}^{-}$ tano in 1994, laparoscopic distal gastrectomy (LDG) has been the standard treatment for gastric cancer. Successful clinical trials that showed the clinical benefits of laparoscopic gastrectomy over open surgery have been published over last 10 years. $^{2}$ The most important advantages of laparoscopic gastrectomy are the minimal invasiveness, including less postoperative pain, shorter recovery, and minimal complications. ${ }^{3}$ Despite these efforts, laparoscopic total gastrectomy has not been as popular as distal gastrectomy, even in recent days. This is partly because of the complexity of a lymph node dissection around the splenic hilum (lymph node \#10), distal splenic artery (lymph node \#11d), and diversity of reconstruction. ${ }^{4}$ Although the learning period of a complete lymph node dissection has been reduced with the continuous training of surgeons, the esophago-jejunal anastomosis procedure is still challenging. Many surgeons have shown various types of anastomosis techniques, but the debate regarding which method is better is ongoing.

Remnant gastric cancer has been used widely to refer to all cancers arising in the residual stomach after a gastrectomy for either benign or malignant disease. ${ }^{5}$ This condition is relatively rare, even in Korea and Japan; however, the incidence has been increased gradually because of the high detection rate of early gastric cancer. Yamada et al. first reported laparoscopic total gastrectomy for remnant gastrectomy in $2005^{6}{ }^{6}$ and several sporadic reports have been published since then.,8 The three points that are critical for safe operation are the adequate lymph node dissection, meticulous adhesiolysis, and reconstruction. ${ }^{9}$ After radical surgery for gastric cancer, the 
intra-abdominal condition is greatly changed. Dense adhesion forms between the liver, gastric bed, pancreas, and transverse colon. In addition, the lymphatic anatomy around the stomach is broken and surgeons should be aware of a newly developed lymphatic system to perform an adequate node dissection. An esophago-jejunal reconstruction is still problematic to surgeons because of the fear for leakage.

In this study, the outcome after TLTG was compared with OTG in patients with RGC. Although most clinical factors, including hospital stay and postoperative complications, were not different between the groups, the bowel function recovery was shorter in the TLTG group. Until more evidence can become available, surgeons should consider the laparoscopic approach.

\section{REFERENCES}

1) Kitano S, Iso Y, Moriyama M, Sugimachi K. Laparoscopy-assisted Billroth I gastrectomy. Surg Laparosc Endosc 1994;4:146-148.

2) Kim HH, Hyung WJ, Cho GS, et al. Morbidity and mortality of laparoscopic gastrectomy versus open gastrectomy for gastric cancer: an interim report: a phase III multicenter, prospective, ran- domized Trial (KLASS Trial). Ann Surg 2010;251:417-420.

3) Song KY, Kim SN, Park CH. Laparoscopy-assisted distal gastrectomy with D2 lymph node dissection for gastric cancer: technical and oncologic aspects. Surg Endosc 2008;22:655-659.

4) Shim JH, Yoo HM, Oh SI, et al. Various types of intracorporeal esophagojejunostomy after laparoscopic total gastrectomy for gastric cancer. Gastric Cancer 2013;16:420-427.

5) Kodera Y, Yamamura Y, Torii A, et al. Gastric remnant carcinoma after partial gastrectomy for benign and malignant gastric lesions. J Am Coll Surg 1996;182:1-6.

6) Yamada H, Kojima K, Yamashita T, Kawano T, Sugihara K, Nihei Z. Laparoscopy-assisted resection of gastric remnant cancer. Surg Laparosc Endosc Percutan Tech 2005;15:226-229.

7) Kwon IG, Cho I, Guner A, et al. Minimally invasive surgery for remnant gastric cancer: a comparison with open surgery. Surg Endosc 2014;28:2452-2458.

8) Nagai E, Nakata K, Ohuchida K, Miyasaka Y, Shimizu S, Tanaka M. Laparoscopic total gastrectomy for remnant gastric cancer: feasibility study. Surg Endosc 2014;28:289-296.

9) Tsunoda S, Okabe H, Tanaka E, et al. Laparoscopic gastrectomy for remnant gastric cancer: a comprehensive review and case series. Gastric Cancer 2016;19:287-292. 